TRANSACTIONS OF THE

AMERICAN MATHEMATICAL SOCIETY

Volume 364, Number 2, February 2012, Pages 1067-1088

S 0002-9947(2011)05491-7

Article electronically published on September 15, 2011

\title{
A TUTTE POLYNOMIAL FOR TORIC ARRANGEMENTS
}

\author{
LUCA MOCI
}

\begin{abstract}
We introduce a multiplicity Tutte polynomial $M(x, y)$, with applications to zonotopes and toric arrangements. We prove that $M(x, y)$ satisfies a deletion-restriction recursion and has positive coefficients. The characteristic polynomial and the Poincaré polynomial of a toric arrangement are shown to be specializations of the associated polynomial $M(x, y)$, likewise the corresponding polynomials for a hyperplane arrangement are specializations of the ordinary Tutte polynomial. Furthermore, $M(1, y)$ is the Hilbert series of the related discrete Dahmen-Micchelli space, while $M(x, 1)$ computes the volume and the number of integer points of the associated zonotope.
\end{abstract}

Ad Alessandro Pucci, che ha ripreso in mano il timone della propria vita.

\section{INTRODUCTION}

The Tutte polynomial is an invariant naturally associated to a matroid and encoding many of its features, such as the number of bases and their internal and external activity ([25, [5, 9]). If the matroid is realized by a finite list of vectors, it is natural to consider the arrangement obtained by taking the hyperplane orthogonal to each vector. One associates to the poset of the intersections of the hyperplanes its characteristic polynomial, which provides a rich combinatorial and topological description of the arrangement (22], [26]). This polynomial can be obtained as a specialization of the Tutte polynomial.

Given a complex torus $T=\left(\mathbb{C}^{*}\right)^{n}$ and a finite list $X$ of characters, i.e. elements of $\operatorname{Hom}\left(T, \mathbb{C}^{*}\right)$, we consider the arrangement of hypersurfaces in $T$ obtained by taking the kernel of each element of $X$. To understand the geometry of this toric arrangement one needs to describe the poset $\mathcal{C}(X)$ of the layers, i.e. connected components of the intersections of the hypersurfaces ([8, [12, [19, 21]). Clearly this poset also depends on the arithmetics of $X$, and not only on its linear algebra: for example, the kernel of the identity character $\lambda$ of $\mathbb{C}^{*}$ is the point $t=1$, but the kernel of $2 \lambda$ has equation $t^{2}=1$, hence is made of two points. Therefore we have no chance to get the characteristic polynomial of $\mathcal{C}(X)$ as a specialization of the ordinary Tutte polynomial $T_{X}(x, y)$ of $X$. In this paper we define a polynomial $M_{X}(x, y)$ that specializes to the characteristic polynomial of $\mathcal{C}(X)$ (Theorem 5.6) and to the Poincaré polynomial of the complement $\mathcal{R}_{X}$ of the toric arrangement (Theorem 5.11). In particular, $M_{X}(1,0)$ is equal to the Euler characteristic of

Received by the editors June 30, 2010 and, in revised form, October 14, 2010.

2010 Mathematics Subject Classification. Primary 52C35; Secondary 05B35, 20G20.

(C)2011 American Mathematical Society

Reverts to public domain 28 years from publication 
$\mathcal{R}_{X}$ and also to the number of connected components of the complement of the arrangement in the compact torus $\bar{T}=\left(\mathbb{S}^{1}\right)^{n}$.

We call $M_{X}(x, y)$ the multiplicity Tutte polynomial of $X$, since it coincides with $T_{X}(x, y)$ when $\mathrm{X}$ is unimodular and, in general, it satisfies the same deletionrestriction recursion that holds for $T_{X}(x, y)$. By this formula (Theorem 3.4) we prove that $M_{X}(x, y)$ has positive coefficients (Theorem 3.5). We leave open the problem of explaining the meaning of these coefficients (Problem 3.7).

A similar polynomial can be defined more generally for matroids if we enrich their structure in order to encode some "arithmetic data"; we call such objects multiplicity matroids. We hope to develop in future papers an axiomatic theory of these matroids, as well as applications to graph theory, which are only outlined here. The focus of the present paper is on the case in which we have a list $X$ of vectors with integer coordinates.

Given such a list, we consider two finite dimensional vector spaces: a space of polynomials $D(X)$ defined by differential equations, and a space of quasi-polynomials $D M(X)$ defined by difference equations. These spaces were introduced by Dahmen and Micchelli to study box splines and partition functions, and are deeply related respectively with the hyperplane arrangement and the toric arrangement defined by $X$, as explained in the recent book 9 . In particular, $T_{X}(1, y)$ is known to be the Hilbert series of $D(X)$, and we will show that $M_{X}(1, y)$ is the Hilbert series of $D M(X)$ (Theorem 6.3).

On the other hand, by Theorem 4.1 the coefficients of $M_{X}(x, 1)$ count integer points in some faces of a convex polytope, the zonotope defined by $X$. The relations between zonotopes and Dahmen-Micchelli spaces has been studied intensively in recent years, giving rise to many algebraic and combinatorial constructions (see in particular, 9], 13, [10, 2], 14, 18]). In particular, $M_{X}(1,1)$ is equal to the volume of the zonotope (Proposition 2.2), while $M_{X}(2,1)$ is the number of its integer points (Proposition 4.5).

Finally, we focus on the case in which $X$ is a root system. We will then show some connections with the theory of Weyl groups (see for instance Corollary 7.4).

\section{Multiplicity Matroids AND MULTIPLICITY TUTTE POLYNOMIALS}

2.1. Definitions. We start by recalling the notions we will be generalizing.

A matroid $\mathfrak{M}$ is a pair $(X, I)$, where $X$ is a finite set and $I$ is a family of subsets of $X$ (called the independent sets) with the following properties:

(1) the empty set is independent.

(2) Every subset of an independent set is independent.

(3) Let $A$ and $B$ be two independent sets and assume that $A$ has more elements than $B$. Then there exists an element $a \in A \backslash B$ such that $B \cup\{a\}$ is still independent.

A maximal independent set is called a basis. The last axiom implies that all bases have the same cardinality, which is called the rank of the matroid. Every $A \subseteq X$ has a natural structure of a matroid, defined by considering a subset of $A$ to be independent if and only if it is in $I$. Then each $A \subseteq X$ has a rank which we denote by $r(A)$. 
The Tutte polynomial of the matroid is then defined as

$$
T(x, y) \doteq \sum_{A \subseteq X}(x-1)^{r(X)-r(A)}(y-1)^{|A|-r(A)} .
$$

From the definition it is clear that $T(1,1)$ is equal to the number of bases of the matroid.

The interested reader may refer for instance to [23, 9].

In the next sections we will recall the two most important examples of matroids and some properties of their Tutte polynomials.

We now introduce the following definitions.

A multiplicity matroid $\mathfrak{M}$ is a triple $(X, I, m)$, where $(X, I)$ is a matroid and $m$ is a function (called multiplicity) from the family of all subsets of $X$ to the positive integers.

We say that $m$ is the trivial multiplicity if it is identically equal to 1 .

We define the multiplicity Tutte polynomial of a multiplicity matroid as

$$
M(x, y) \doteq \sum_{A \subseteq X} m(A)(x-1)^{r(X)-r(A)}(y-1)^{|A|-r(A)} .
$$

Let us remark that we can endow every matroid with the trivial multiplicity, and then $M(x, y)=T(x, y)$.

Remark 2.1. Given any two matroids $\mathfrak{M}_{1}=\left(X_{1}, I_{1}\right)$ and $\mathfrak{M}_{2}=\left(X_{2}, I_{2}\right)$, we can naturally define a matroid $\mathfrak{M}_{1} \oplus \mathfrak{M}_{2}=(X, I)$, where $X$ is the disjoint union of $X_{1}$ and $X_{2}$, and $A \in I$ if and only if $A_{1} \doteq A \cap X_{1} \in I_{1}$ and $A_{2} \doteq A \cap X_{2} \in I_{2}$. Moreover, if $\mathfrak{M}_{1}$ and $\mathfrak{M}_{2}$ have multiplicity functions $m_{1}$ and $m_{2}$, then $m(A) \doteq m_{1}\left(A_{1}\right) \cdot m_{2}\left(A_{2}\right)$ defines a multiplicity on $\mathfrak{M}_{1} \oplus \mathfrak{M}_{2}$. We notice that the rank of a subset $A$ is just the sum of the ranks of $A_{1}$ and $A_{2}$, and so it is easily seen that the (multiplicity) Tutte polynomial of $\mathfrak{M}_{1} \oplus \mathfrak{M}_{2}$ is the product of the (multiplicity) Tutte polynomials of $\mathfrak{M}_{1}$ and $\mathfrak{M}_{2}$.

2.2. Lists of vectors and zonotopes. Let $X$ be a finite list of vectors spanning a real vector space $U$, and let $I$ be the family of its linearly independent subsets. Then $(X, I)$ is a matroid, and the rank of a subset $A$ is just the dimension of the spanned subspace. We denote by $T_{X}(x, y)$ the associated Tutte polynomial.

We to associate the list $X$ a zonotope, that is, a convex polytope in $U$ defined as follows:

$$
\mathcal{Z}(X) \doteq\left\{\sum_{x \in X} t_{x} x, 0 \leq t_{x} \leq 1\right\}
$$

Zonotopes play an important role in the theory of hyperplane arrangements, and also in that of splines, a class of functions studied in Approximation Theory (see [9]).

We recall that a lattice $\Lambda$ of rank $n$ is a discrete subgroup of $\mathbb{R}^{n}$ which spans $\mathbb{R}^{n}$ as a real vector space. Every such $\Lambda$ can be generated from some basis of the vector space by forming all linear combinations with integer coefficients; hence the group $\Lambda$ is isomorphic to $\mathbb{Z}^{n}$. We will always use the term lattice with this meaning, and not in the combinatorial sense (a poset with join and meet).

Now let $X$ be a finite list of elements in a lattice $\Lambda$, and let $I$ and $r$ be as above. For every $A \subset X$, we denote by $\langle A\rangle_{\mathbb{Z}}$ and $\langle A\rangle_{\mathbb{R}}$ respectively the sublattice of $\Lambda$ and 
the subspace of $U \doteq \Lambda \otimes \mathbb{R}$ spanned by $A$. Let us define

$$
\Lambda_{A} \doteq \Lambda \cap\langle A\rangle_{\mathbb{R}}
$$

the largest sublattice of $\Lambda$ in which $\langle A\rangle_{\mathbb{Z}}$ has finite index. We define $m$ as this index:

$$
m(A) \doteq\left[\Lambda_{A}:\langle A\rangle_{\mathbb{Z}}\right] .
$$

This defines a multiplicity matroid and then a multiplicity Tutte polynomial $M_{X}(x, y)$, which is the main subject of this paper.

Concretely, if we identify $\Lambda$ with $\mathbb{Z}^{n}$, we have that for every $A \subset X$ of maximal rank, $m(A)$ is equal to the GCD of the determinants of the bases extracted from A.

We say that the list $X$ is unimodular if every $B \subset X$ that is a basis for $U$ over $\mathbb{R}$ spans $\Lambda$ over $\mathbb{Z}$ (i.e., in the identification above, $B$ has determinant \pm 1 ). In this case the multiplicity is trivial and $M_{X}(x, y)=T_{X}(x, y)$.

We now start by showing some relations with the zonotope $\mathcal{Z}(X)$ generated by $X$ in $U$.

We already observed that $T_{X}(1,1)$ is equal to the number of bases that can be extracted from $X$. On the other hand we have

Proposition 2.2. $M_{X}(1,1)$ is equal to the volume of the zonotope $\mathcal{Z}(X)$.

Proof. By 24, $\mathcal{Z}(X)$ is paved by a family of polytopes $\left\{\Pi_{B}\right\}$, where $B$ varies among all the bases extracted from $X$, and every $\Pi_{B}$ is obtained by translating the zonotope $\mathcal{Z}(B)$ generated by the sublist $B$. Hence

$$
\operatorname{vol}\left(\Pi_{B}\right)=|\operatorname{det}(B)| .
$$

However, when $B$ is a basis,

$$
m(B)=\left[\Lambda:\langle B\rangle_{\mathbb{Z}}\right]=|\operatorname{det}(B)| .
$$

Since

$$
M_{X}(1,1)=\sum_{B \subset X, B \text { basis }} m(B)
$$

the claim follows.

Further relations between the polynomial $M_{X}(x, y)$ and the zonotope $\mathcal{Z}(X)$ will be shown in Section 4

2.3. Graphs. Let $G$ be a finite graph, $V(G)$ be the set of its vertices, and $X$ be the set of its edges. For every $A \subseteq X$, we consider the subgraph of $G$ having set of vertices $V(G)$ and set of edges $A$. By abuse of notation, we denote this subgraph by $A$. We define $I$ as the set of the forests in $G$ (subgraphs whose connected components are simply connected). Then $(X, I)$ is a matroid with rank function

$$
r(A)=|V(G)|-c(A),
$$

where $c(A)$ is the number of connected components of $A$.

Remark 2.3. If $G$ has neither loops nor multiple edges, let us take a vector space $\widetilde{U}$ with basis $e_{1}, \ldots, e_{n}$ bijective to $V(G)$, and associate to the edge connecting two vertices $i$ and $j$ the vector $e_{i}-e_{j}$. In this way we get a list $X_{G}$ of vectors bijective to $X$ and spanning a hyperplane $U$ in $\widetilde{U}$. Since in this bijection the rank is preserved and forests correspond to linearly independent sets, $G$ and $X_{G}$ define the same matroid and have the same Tutte polynomial. 
Now let us assume every edge $e \in X$ to have an integer label $m_{e}>0$. By defining

$$
m(A) \doteq \prod_{e \in A} m_{e}
$$

we get a multiplicity matroid and then a multiplicity Tutte polynomial $M_{G}(x, y)$.

We may view the labels $m_{e}$ as multiplicities of the edges in the following way. Let us define a new graph $G_{m}$ with the same vertices as $G$, but with $m_{e}$ edges connecting the two vertices incident to $e \in X$. Now let $S\left(G_{m}\right)$ be the set of simple subgraphs of $G_{m}$, i.e. subgraphs with at most one edge connecting any two vertices, and at most one loop on every vertex. It is then clear that

$$
M_{G}(x, y) \doteq \sum_{A \in S\left(G_{m}\right)}(x-1)^{r(X)-r(A)}(y-1)^{|A|-r(A)} .
$$

In particular, $M_{G}(2,1)$ is equal to the number of forests of $G_{m}$, and $M_{G}(1,1)$ is the number of spanning trees (i.e., trees connecting all the vertices) of $G_{m}$.

\section{Deletion-RESTRICTION FORMUla AND POSITIVITy}

The central idea that inspired Tutte in defining the polynomial $T(x, y)$ was to find the most general invariant satisfying a recursion known as deletion-restriction. Such a recursion allows us to reduce the computation of the Tutte polynomial to some trivial cases.

In the two examples above (when the matroid is defined by a list of vectors or by a graph), the polynomial $M(x, y)$ satisfies a similar recursion. We will explain and prove the algorithm in the first case. The case of graphs (where the recursion is known as deletion-contraction) is described in [20, Section 2.3.1].

3.1. Lists of vectors. Let $X$ be a finite list of elements spanning a vector space $U$, and let $\lambda \in X$ be a nonzero element. We define two new lists: the list $X_{1} \doteq X \backslash\{\lambda\}$ of elements of $U$ and the list $X_{2}$ of elements of $U /\langle\lambda\rangle$ obtained by reducing $X_{1}$ modulo $\lambda$. Assume that $\lambda$ is dependent in $X$, i.e. $\lambda \in\left\langle X_{1}\right\rangle_{\mathbb{R}}$. Then we have the following well-known formula:

\section{Theorem 3.1.}

$$
T_{X}(x, y)=T_{X_{1}}(x, y)+T_{X_{2}}(x, y) .
$$

It is now clear why we defined $X$ as a list, and not as a set: even if we start with $X$ composed by distinct (nonzero) elements, some vectors in $X_{2}$ may appear many times (and some vectors may be zero).

Notice that by applying the above formula recursively, our problem reduces to computing $T_{Y}(x, y)$ when $Y$ is the union of a list $Y_{1}$ of $k$ linearly independent vectors and of a list $Y_{0}$ of $h$ zero vectors $(k, h \geq 0)$. In this case the Tutte polynomial is easily computed.

\section{Lemma 3.2.}

$$
T_{Y}(x, y)=x^{k} y^{h} .
$$

Proof. Given any $\lambda \in Y_{1}$, since

$$
\langle Y\rangle_{\mathbb{R}}=\langle Y \backslash\{v\}\rangle_{\mathbb{R}} \oplus\langle\{v\}\rangle_{\mathbb{R}},
$$

by Remark 2.1 we have that

$$
T_{Y}(x, y)=x T_{Y \backslash\{\lambda\}}(x, y) .
$$


Hence by induction we get that $T_{Y}=x^{k} T_{Y_{0}}$. Finally

Thus we get:

$$
T_{Y_{0}}(x, y)=\sum_{j=0}^{h}\left(\begin{array}{l}
h \\
j
\end{array}\right)(y-1)^{j}=((y-1)+1)^{h}=y^{h} .
$$

Theorem 3.3. $T_{X}(x, y)$ is a polynomial with positive coefficients.

3.2. Lists of elements in abelian groups. We now want to show a similar recursion for the polynomial $M_{X}(x, y)$. Inspired by [11, we noticed that we need to work in a larger category. Indeed, whereas the quotient of a vector space by a subspace is still a vector space, the quotient of a lattice by a sublattice is not a lattice, but a finitely generated abelian group. For example, in the 1-dimensional case, the quotient of $\mathbb{Z}$ by $m \mathbb{Z}$ is the cyclic group of order $m$.

Then let $\Gamma$ be a finitely generated abelian group. For every subset $S$ of $\Gamma$ we denote by $\langle S\rangle$ the generated subgroup. We recall that $\Gamma$ is isomorphic to the direct product of a lattice $\Lambda$ and of a finite group $\Gamma_{t}$, which is called the torsion subgroup of $\Gamma$. We denote by $\pi$ the projection $\pi: \Gamma \rightarrow \Lambda$.

Let $X$ be a finite subset of $\Gamma$. For every $A \subseteq X$ we set

$$
\Lambda_{A} \doteq \Lambda \cap\langle\pi(A)\rangle_{\mathbb{R}}
$$

and

$$
\Gamma_{A} \doteq \Lambda_{A} \times \Gamma_{t} .
$$

In other words, $\Gamma_{A}$ is the largest subgroup of $\Gamma$ in which $\langle A\rangle$ has finite index.

Now we define

$$
m(A) \doteq\left[\Gamma_{A}:\langle A\rangle\right]
$$

We also define $r(A)$ as the rank of $\pi(A)$. In this way we defined a multiplicity matroid, to which we associate its multiplicity Tutte polynomial:

$$
M_{X}(x, y) \doteq \sum_{A \subseteq X} m(A)(x-1)^{r(X)-r(A)}(y-1)^{|A|-r(A)} .
$$

It is clear that if $\Gamma$ is a lattice, these definitions coincide with those given in the previous sections.

If in the opposite case, $\Gamma$ is a finite group, then $M(x, y)$ is a polynomial in which only the variable $y$ appears. Furthermore, this polynomial evaluated at $y=1$ gives the order of $\Gamma$. Indeed the only summand that does not vanish is the contribution of the empty set, which generates the trivial subgroup.

Now let $\lambda \in X$ be an element such that $\pi(\lambda) \neq 0$ and

We set

$$
\pi(\lambda) \in\langle\pi(X \backslash\{\lambda\})\rangle_{\mathbb{R}} .
$$

$$
X_{1} \doteq X \backslash\{\lambda\} \subset \Gamma .
$$

For every $A \subseteq X$, we denote by $\bar{A}$ its image under the natural projection

$$
\Gamma \longrightarrow \Gamma /\langle\lambda\rangle \text {. }
$$

Since $\Gamma /\langle\lambda\rangle$ is a finitely generated abelian group and $\bar{A}$ is a subset of it, $m(\bar{A})$ is defined. Notice that

$$
m(\bar{A}) \doteq\left[(\Gamma /\langle\lambda\rangle)_{\bar{A}}:\langle\bar{A}\rangle\right]=\left[\Gamma_{A} /\langle\lambda\rangle:\langle A\rangle /\langle\lambda\rangle\right]=\left[\Gamma_{A}:\langle A\rangle\right]=m(A) .
$$

We denote by $X_{2}$ the subset $\overline{X_{1}}$ of $\Gamma /\langle\lambda\rangle$. We have the following deletion-restriction formula. 


\section{Theorem 3.4.}

$$
M_{X}(x, y)=M_{X_{1}}(x, y)+M_{X_{2}}(x, y) .
$$

Proof. The sum expressing $M_{X}(x, y)$ splits into two parts. The first is over the sets $A \subseteq X_{1}$,

$$
\sum_{A \subseteq X_{1}} m(A)(x-1)^{r(X)-r(A)}(y-1)^{|A|-r(A)}=M_{X_{1}}(x, y),
$$

since clearly $r(X)=r\left(X_{1}\right)$. The second part is over the sets $A$ such that $\lambda \in A$. For such sets we have that

$$
|\bar{A}|=|A|-1, r(\bar{A})=r(A)-1, r\left(X_{2}\right)=r(X)-1, m(\bar{A})=m(A) .
$$

Therefore

$$
\begin{aligned}
& \sum_{A \subseteq X, \lambda \in A} m(A)(x-1)^{r(X)-r(A)}(y-1)^{|A|-r(A)} \\
& =\sum_{\bar{A} \subseteq X_{2}} m(\bar{A})(x-1)^{r\left(X_{2}\right)-r(\bar{A})}(y-1)^{|\bar{A}|-r(\bar{A})}=M_{X_{2}}(x, y) .
\end{aligned}
$$

Now we can prove

Theorem 3.5. $M_{X}(x, y)$ is a polynomial with positive coefficients.

Proof. By applying recursively the formula above, we need only consider lists that do not contain any $\lambda$ satisfying condition (2). Any such list $Y$ is made of elements of some quotient $\Gamma(Y)$ of $\Gamma$, and we can assume $Y$ to be the disjoint union of a list $Y_{0}$ of $h$ zeros $(h \geq 0)$, and of a list $Y_{1}$ such that $\pi\left(Y_{1}\right)$ is a basis of $\Lambda(Y) \otimes \mathbb{R}$. (Here we denote by $\pi$ the projection $\Gamma(Y) \rightarrow \Lambda(Y)$, where $\Gamma(Y) \simeq \Lambda(Y) \times \Gamma(Y)_{t}$ is the product of the lattice and of the torsion subgroup.) We first notice that

$$
M_{Y_{0}}=\left|\Gamma(Y)_{t}\right| \sum_{j=0}^{h}\left(\begin{array}{c}
h \\
j
\end{array}\right)(y-1)^{j}=\left|\Gamma(Y)_{t}\right|((y-1)+1)^{h}=\left|\Gamma(Y)_{t}\right| y^{h} .
$$

Furthermore, it is easily seen that

$$
M_{Y}(x, y)=M_{Y_{0}}(x, y) M_{Y_{1}}(x, y) .
$$

Finally, the positivity of $M_{Y_{1}}(x, y)$ will be proved in Lemma 4.4 .

3.3. Statistics. Usually, polynomials with positive coefficients encode some statistics. In other words, their coefficients count something.

For example, the Tutte polynomial embodies two statistics on the set of the bases called internal and external activity. Although they can be stated for an abstract matroid (see for example [9, Section 2.2.2]), we give such definitions for a list $X$ of vectors. Let $B$ be a basis extracted from $X$.

(1) We say that $v \in X \backslash B$ is externally active for $B$ if $v$ is a linear combination of the elements of $B$ following it (in the total ordering fixed on $X$ ).

(2) We say that $v \in B$ is internally active for $B$ if there is no element $w$ in $X$ preceding $v$ such that $\{w\} \cup(B \backslash\{v\})$ is a basis.

(3) The number $e(B)$ of externally active elements is called the external activity of $B$.

(4) The number $i(B)$ of internally active elements is called the internal activity of $B$. 
The following result is proved in [5]:

Theorem 3.6.

$$
T_{X}(x, y)=\sum_{B \subseteq X, B \text { basis }} x^{i(B)} y^{e(B)} .
$$

Hence the coefficients of $T_{X}(x, y)$ count the number of bases having a given internal and external activity.

Since $M_{X}(x, y)$ has positive coefficients too, it is natural to wonder what the statistics involved are.

Problem 3.7. Give a combinatorial interpretation of the coefficients of $M_{X}(x, y)$.

Although we leave this question open, in Theorems 4.1 and 6.3 we show the meaning of the coefficients of $M_{X}(x, 1)$ and $M_{X}(1, y)$ respectively.

We say that a basis $B$ of $X$ is a no-broken circuit if $e(B)=0$. We denote by $n b c(X)$ the number of no-broken circuit bases of $X$. It is clear from Theorem 3.6 that

$$
n b c(X)=T_{X}(1,0) .
$$

We will use this formula in the following sections.

\section{INTEGER POINTS IN ZONOTOPES}

Let $X$ be a finite list of vectors contained in a lattice $\Lambda$ and generating the vector space $U=\Lambda \otimes \mathbb{R}$. We say that a point of $U$ is integer if it is contained in $\Lambda$. In this section we prove that $M_{X}(2,1)$ is equal to the number of integer points of the zonotope $\mathcal{Z}(X)$. Moreover, we compare this number with the volume. To do this, we have to move the zonotope to a "generic position".

Following [9, Section 1.3], we define the cut-locus of the couple $(\Lambda, X)$ as the union of all hyperplanes in $U$ that are translations, under elements of $\Lambda$, of the linear hyperplanes spanned by subsets of $X$. Let $\underline{\varepsilon}$ be a vector of $U$ that does not lie in the cut-locus and has length $\varepsilon \ll 1$. Let $\mathcal{Z}(X)-\varepsilon$ be the polytope obtained by translating $\mathcal{Z}(X)$ by $-\underline{\varepsilon}$, and let $\Im(X)$ be the set of its integer points:

$$
\Im(X) \doteq(\mathcal{Z}(X)-\underline{\varepsilon}) \cap \Lambda .
$$

It is intuitive (and proved in [9, Prop. 2.50]) that this number is equal to the volume:

$$
|\Im(X)|=\operatorname{vol}(\mathcal{Z}(X))=M_{X}(1,1)
$$

by Proposition 2.2. We now prove a stronger result.

Let us choose $\underline{\varepsilon}$ so that $\mathcal{Z}(X)-\underline{\varepsilon}$ contains the origin $\underline{0}$. We partition $\mathfrak{I}(X)$ as follows: set $\mathfrak{I}_{n}(X)=\{\underline{0}\}$, and for every $k=n-1, \ldots, 0$, let $\mathfrak{I}_{k}(X)$ be the set of elements of $\mathfrak{I}(X)$ that are contained in some $k$-codimensional face of $\mathcal{Z}(X)$ and that are not contained in $\mathfrak{I}_{h}(X)$ for $h>k$. Then we have:

\section{Theorem 4.1.}

$$
M_{X}(x, 1)=\sum_{k=0}^{n}\left|\Im_{k}(X)\right| x^{k} .
$$

Example 4.2. Consider the list in $\mathbb{Z}^{2}$ :

$$
X=\{(3,3),(1,-1),(2,0)\} .
$$




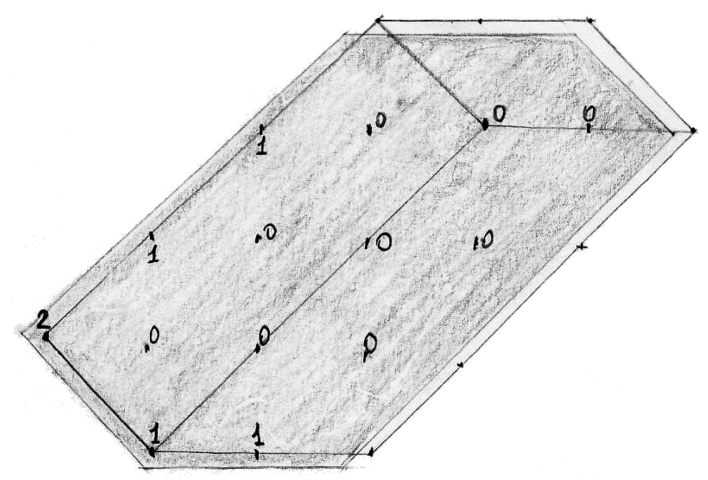

FiguRE 1

Then

$$
M_{X}(x, y)=(x-1)^{2}+(3+1+2)(x-1)+(6+6+2)+2(y-1) .
$$

Hence

$$
M_{X}(x, 1)=x^{2}+4 x+9
$$

and $M_{X}(2,1)=21$. Indeed the zonotope $\mathcal{Z}(X)$ has area 14 and contains 21 integer points, 14 of which lie in $\mathcal{Z}(X)-\underline{\varepsilon}$, represented by the shaded portion in Figure 1. The sets $\mathfrak{I}_{2}(X), \mathfrak{I}_{1}(X)$, and $\mathfrak{I}_{0}(X)$ contain 1,4 and 9 points, respectively, each marked by the subscript of its set.

In order to prove Theorem 4.1, we first assume $X$ to be linearly independent (and hence a basis for $U$ ). In this case the zonotope is called a parallelepiped. In particular, for every $A \subseteq X$ the zonotope $\mathcal{Z}(A)$ is a face of $\mathcal{Z}(X)$. Moreover, we can choose $\underline{\varepsilon}$ so that the faces of $\mathcal{Z}(X)$ whose interior is contained in $\mathcal{Z}(X)-\underline{\varepsilon}$ are precisely those of type $\mathcal{Z}(A)$ for some $A \subseteq X$ : for instance take

$$
\underline{\varepsilon}=\sum_{\lambda \in X} \frac{\varepsilon}{n} \lambda \text {. }
$$

We say that an integer point is internal to a face of $\mathcal{Z}(X)$ if that face is the smallest face containing this point. We denote by $h(A)$ the number of integer points that are internal to $\mathcal{Z}(A)$.

Lemma 4.3. For every $A \subset X$,

$$
h(A)=\sum_{B \subseteq A}(-1)^{|A|-|B|} m(B) .
$$

Proof. By construction, $\mathcal{Z}(X)-\underline{\varepsilon}$ contains exactly the integer points that are internal to the faces $\mathcal{Z}(A), A \subseteq X$. Hence

$$
|\Im(X)|=\sum_{A \subseteq X} h(A) .
$$

Moreover, by Formula (1), $m(X)$ is equal to the volume of $\mathcal{Z}(X)$. Thus we proved:

$$
m(X)=\sum_{A \subseteq X} h(A) .
$$


We get the claim by the inclusion-exclusion principle, since the intersection of two faces $\mathcal{Z}\left(A_{1}\right), \mathcal{Z}\left(A_{2}\right)$ is the face $\mathcal{Z}\left(A_{1} \cap A_{2}\right)$.

Now we prove

Lemma 4.4. Let $X$ be a basis for $U$. Then

$$
M_{X}(x, 1)=\sum_{k=0}^{n}\left(\sum_{A \subseteq X,|A|=n-k} h(A)\right) x^{k} .
$$

Proof. By definition,

$$
M_{X}(x, y)=\sum_{A \subseteq X} m(A)(x-1)^{n-|A|} .
$$

The coefficient of $x^{k}$ in this expression is

$$
\sum_{A \subseteq X,|A| \leq n-k}(-1)^{n-k-|A|}\left(\begin{array}{c}
n-|A| \\
k
\end{array}\right) m(A) .
$$

By the previous lemma, our claim amounts to proving that the coefficient of $x^{k}$ is

$$
\sum_{A \subseteq X,|A|=n-k} \sum_{B \subseteq A}(-1)^{|A|-|B|} m(B)=\sum_{B \subseteq X,|B| \leq n-k}(-1)^{n-k-|B|}\left(\begin{array}{c}
n-|B| \\
k
\end{array}\right) m(B)
$$

because every $B \subseteq X$ is contained in exactly

$$
\left(\begin{array}{c}
n-|B| \\
n-k-|B|
\end{array}\right)=\left(\begin{array}{c}
n-|B| \\
k
\end{array}\right)
$$

sets $A \subseteq X$ of cardinality $n-k$.

In this way the theorem is proved for a linearly independent set of vectors, since

$$
\left|\mathfrak{I}_{k}(X)\right|=\sum_{A \subseteq X,|A|=n-k} h(A) .
$$

As in Section [3.2, given a nonzero element $\lambda \in X$ we set $X_{1} \doteq X \backslash\{\lambda\}$ and we denote by $X_{2}$ the image of $X_{1}$ under the natural projection

$$
p: U \longrightarrow U /\langle\lambda\rangle_{\mathbb{R}} .
$$

Set $m_{\lambda} \doteq m(\{\lambda\})=\left[\Lambda \cap\langle\lambda\rangle_{\mathbb{R}}:\langle\lambda\rangle_{\mathbb{Z}}\right]$. Note that $X_{1}$ defines a zonotope $\mathcal{Z}\left(X_{1}\right) \subset$ $\mathcal{Z}(X)$, and $X_{2}$ defines a zonotope $\mathcal{Z}\left(X_{2}\right)$ in the quotient space $p(U)$. We briefly recall some results from [9, Section 2.3]. We suggest that the reader look at Figure 2 (in which $\mathcal{Z}\left(X_{1}\right)$ is the white rectangle, and $\lambda$ is the vector of coordinates $(2,0)$ ). The 1-parameter group of translations $u \mapsto u+t \lambda$, acting on $U$, has orbits that are the fibers of $p$. For every $u \in \mathcal{Z}\left(X_{2}\right)$, let $s(u)$ be the point $u+t \lambda$ where the translation group "exits" the zonotope $Z\left(X_{1}\right)$. Then $s\left(\mathcal{Z}\left(X_{2}\right)\right)$ (the bold line in the picture) is a piece of the boundary of $\mathcal{Z}\left(X_{1}\right)$ naturally identified to $\mathcal{Z}\left(X_{2}\right)$. Furthermore, we have the following decomposition:

$$
\mathcal{Z}(X)=\mathcal{Z}\left(X_{1}\right) \cup \widetilde{\mathcal{Z}}\left(X_{2}\right)
$$

where $\mathcal{Z}\left(X_{1}\right) \cap \widetilde{\mathcal{Z}}\left(X_{2}\right)=s\left(\mathcal{Z}\left(X_{2}\right)\right)$, and the polytope $\widetilde{\mathcal{Z}}\left(X_{2}\right)$ (shaded in the picture) is a product of the polytope $s\left(\mathcal{Z}\left(X_{2}\right)\right)$ and the segment $\left[0, m_{\lambda}\right]$. So $p$ maps $\widetilde{\mathcal{Z}}\left(X_{2}\right)$ on $\mathcal{Z}\left(X_{2}\right)$ with fiber $\left[0, m_{\lambda}\right]$ ([9, Section 2.3]).

We can now conclude the proof of Theorem 4.1 . 


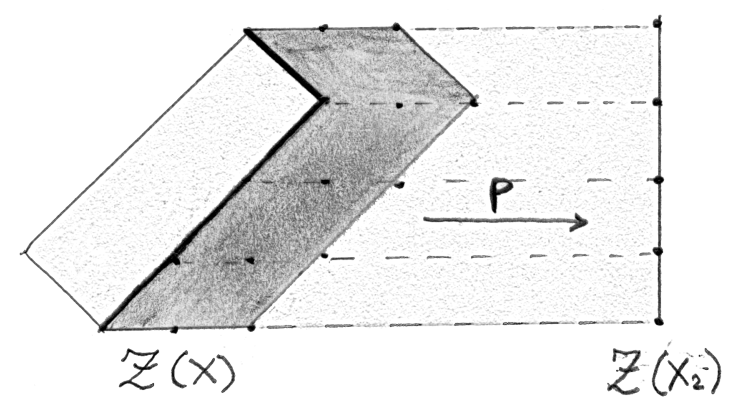

Figure 2

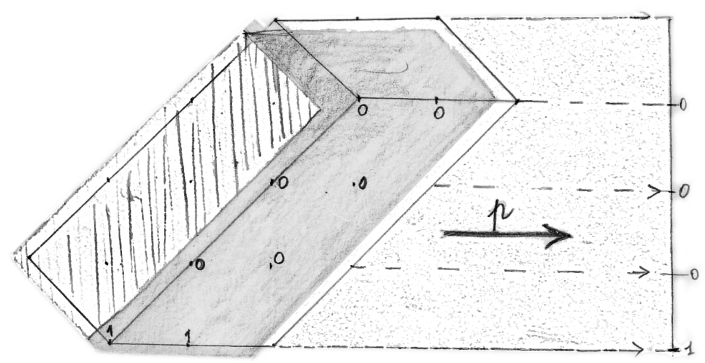

FiguRE 3

Proof. We can assume that $X$ contains only nonzero elements. If $X$ is independent, we have Lemma 4.4. If $X$ has rank 0 , the statement is trivial. Thus we assume $X$ to be dependent and of positive rank, we choose a vector $\lambda$ that lies in the cone spanned by the other vectors, and we proceed inductively by deletion-restriction. We notice that the restriction of $p$ to $\mathfrak{I}_{k}(X) \backslash \mathfrak{I}_{k}\left(X_{1}\right)$ maps this set onto $\mathfrak{I}_{k}\left(X_{2}\right)$, and the fiber of every point has cardinality $m_{\lambda}$. (See Figure 3 .) Thus

$$
\left|\mathfrak{I}_{k}(X)\right|=\left|\mathfrak{I}_{k}\left(X_{1}\right)\right|+m_{\lambda}\left|\mathfrak{I}_{k}\left(X_{2}\right)\right|=M_{X_{1}}(x, 1)+M_{X_{2}}(x, 1)
$$

by the inductive hypothesis, since $m_{\lambda}$ is the torsion of $\Lambda /\langle\lambda\rangle_{\mathbb{Z}}$. Hence the claim follows by Theorem 3.4 .

In the same way we can prove

Proposition 4.5. The number $|\mathcal{Z}(X) \cap \Lambda|$ of integer points in the zonotope is equal to $M_{X}(2,1)$.

Proof. By applying deletion-restriction as in the previous proof, we can reduce to the case in which $X$ is a basis of $U$. Then in this basis, $\mathcal{Z}(X)$ is a parallelepiped. For every face $F$ we define $A_{F}$ as the subset of $X$ corresponding to the coordinates which are not constant on $F$. Since all the other coordinates are identically equal either to 0 or to 1 , for every $A \subseteq X$ there are exactly $2^{k}$ faces $F$ such that $A_{F}=A$, $k=|X \backslash A|$. Among these faces, the only one contributing to $M_{X}(1,1)$ is the 
one whose constant coordinates are all equal to 0, i.e., $Z(A)$. On the other hand, to compute the total number of integer points we have to take all these $2^{k}$ faces. Since any two of them are disjoint and contain the same number of points in their interior, by Theorem 4.1 we get the claim.

\section{Application to ARRAngements}

In this section we describe some geometrical objects related to the lists considered in Section 2.2, and we show that many of their features are encoded in the polynomials $T_{X}(x, y)$ and $M_{X}(x, y)$.

5.1. Recall on hyperplane arrangements. Let $X$ be a finite list of elements of a vector space $U$. A hyperplane arrangement $\mathcal{H}(X)$ is defined in the dual space $V=$ $U^{*}$, by taking the orthogonal hyperplane of each element of $X$. Conversely, given an arrangement of hyperplanes in a vector space $V$, let us choose for each hyperplane a nonzero vector in $V^{*}$ orthogonal to it. Let $X$ be the list of such vectors. Since every element of $X$ is determined up to scalar multiples, the matroid associated to $X$ is well defined. In this way a Tutte polynomial is naturally associated to the hyperplane arrangement.

The importance of the Tutte polynomial in the theory of hyperplane arrangements is well known. Here we recall some results which will be generalized in the next sections.

To every sublist $A \subseteq X$ we associate the subspace $A^{\perp}$ of $V$ that is the intersection of the corresponding hyperplanes of $\mathcal{H}(X)$. In other words, $A^{\perp}$ is the subspace of vectors that are orthogonal to every element of $A$. Let $\mathcal{L}(X)$ be the set of such subspaces, partially ordered by reverse inclusion, and having as minimal element (which we denote by $\mathbf{0}$ ) the whole space $V=\emptyset^{\perp} \cdot \mathcal{L}(X)$ is called the intersection poset of the arrangement and is the main combinatorial object associated to a hyperplane arrangement.

We also recall that to every finite poset $\mathcal{P}$ there is an associated Moebius function

$$
\mu: \mathcal{P} \times \mathcal{P} \rightarrow \mathbb{Z}
$$

recursively defined as follows:

$$
\mu(L, M)= \begin{cases}0 & \text { if } L>M \\ 1 & \text { if } L=M \\ -\sum_{L \leq N<M} \mu(L, N) & \text { if } L<M\end{cases}
$$

Notice that the poset $\mathcal{L}(X)$ is ranked by the dimension of the subspaces. We define the characteristic polynomial of the poset as

$$
\chi(q) \doteq \sum_{L \in \mathcal{L}(X)} \mu(\mathbf{0}, L) q^{\operatorname{dim}(L)} .
$$

This is an important invariant of $\mathcal{H}(X)$. Indeed, let $\mathcal{M}_{X}$ be the complement in $V$ of the union of the hyperplanes of $\mathcal{H}(X)$. Let $P(q)$ be Poincaré polynomial of $\mathcal{M}_{X}$, i.e. the polynomial having the $k$-th Betti number of $\mathcal{M}_{X}$ as the coefficient of $q^{k}$. If $V$ is a complex vector space, by [22] we have the following theorem. 


\section{Theorem 5.1.}

$$
P(q)=(-q)^{n} \chi(-1 / q) .
$$

If, on the other hand, $V$ is a real vector space, by [26] the number $C h(X)$ of chambers (i.e., connected components of $\mathcal{M}_{X}$ ) is:

\section{Theorem 5.2.}

$$
C h(X)=(-1)^{n} \chi(-1) .
$$

The Tutte polynomial $T_{X}(x, y)$ turns out to be a stronger invariant, in the following sense. Assume that $\underline{0} \notin X$; then

\section{Theorem 5.3.}

$$
(-1)^{n} T_{X}(1-q, 0)=\chi(q) .
$$

The proof of these theorems can be found, for example, in 9, Theorems 10.5, 2.34 and 2.33].

Notice that in the present section we have only considered central arrangements, i.e. arrangements in which all the hyperplanes contain the origin. More generally, one may consider arrangements of affine hyperplanes in affine spaces. In this setting, Ardila defined a notion of a semimatroid and a Tutte polynomial (see [1]).

5.2. Toric arrangements and their generalizations. Let $\Gamma=\Lambda \times \Gamma_{t}$ be a finitely generated abelian group and define

$$
T_{\Gamma} \doteq \operatorname{Hom}\left(\Gamma, \mathbb{C}^{*}\right) .
$$

$T_{\Gamma}$ has a natural structure of an abelian linear algebraic group: indeed, it is the direct product of a complex torus $T_{\Lambda}$ of the same rank as $\Lambda$ and of the finite group $\Gamma_{t}^{*}$ dual to $\Gamma_{t}$ (and isomorphic to it).

Moreover, $\Gamma$ is identified with the group of characters of $T_{\Gamma}$ : indeed, given $\lambda \in \Lambda$ and $t \in T_{\Gamma}$, we can take any representative $\varphi_{t} \in \operatorname{Hom}(\Gamma, \mathbb{C})$ of $t$ and set

$$
\lambda(t) \doteq e^{2 \pi i \varphi_{t}(\lambda)} .
$$

When this is not ambiguous we will denote $T_{\Gamma}$ by $T$.

Let $X \subset \Lambda$ be a finite subset spanning a sublattice of $\Lambda$ of finite index. The kernel of every character $\chi \in X$ is a (nonconnected) subvariety of $T$ :

$$
H_{\chi} \doteq\{t \in T \mid \chi(t)=1\} \text {. }
$$

The collection $\mathcal{T}(X)=\left\{H_{\chi}, \chi \in X\right\}$ is called the generalized toric arrangement defined by $X$ on $T$.

We denote by $\mathcal{R}_{X}$ the complement of the arrangement:

$$
\mathcal{R}_{X} \doteq T \backslash \bigcup_{\chi \in X} H_{\chi}
$$

and by $\mathcal{C}_{X}$ the set of all the connected components of all the intersections of the subvarieties $H_{\chi}$, ordered by reverse inclusion and having as minimal elements the connected components of $T$.

Since $\operatorname{rank}(\Lambda)=\operatorname{dim}(T)$, the maximal elements of $\mathcal{C}(X)$ are 0-dimensional; hence (since they are connected) they are points. We denote by $\mathcal{C}_{0}(X)$ the set of such layers, which we call the points of the arrangement.

Given $A \subseteq X$ let us define

$$
H_{A} \doteq \bigcap_{\lambda \in A} H_{\lambda}
$$


Lemma 5.4. $m(A)$ is equal to the number of connected components of $H_{A}$.

Proof. It is clear by definition that $m(X)=\left|H_{X}\right|$. Then for every $A \subseteq X$, we have that

$$
\left|\overline{H_{A}}\right|=m(A),
$$

where $\overline{H_{A}}$ is the set of the points of the arrangement $\mathcal{T}(A)$ defined by $A$ in $T_{\Gamma_{A}}$. Now let $H_{A}{ }^{0}$ be the connected component of $H_{A}$ that contains the identity. This is a subtorus of $T_{\Gamma}$, and the quotient map

$$
T_{\Gamma} \rightarrow T_{\Gamma} / H_{A}{ }^{0} \simeq T_{\Gamma_{A}}
$$

induces a bijection between the connected components of $H_{A}$ and the points of $\overline{H_{A}}$.

In particular, when $\Gamma$ is a lattice, $T$ is a torus and $\mathcal{T}(X)$ is called the toric arrangement defined by $X$. Such arrangements have been studied for example in [17, 8], 19], 21]; see [9] for a complete reference. In particular, the complement $\mathcal{R}_{X}$ has been described topologically and geometrically. In this description the poset $\mathcal{C}(X)$ plays a major role; for many aspects analogous to that of the intersection poset for hyperplane arrangements, see [8], 21].

We now explain the importance in this framework of the polynomial $M_{X}(x, y)$ defined in Section 3.3.

5.3. Characteristic polynomial. Let $\mu$ be the Moebius function of $\mathcal{C}(X)$. Notice that we have a natural rank function given by the dimension of the layers. For every $C \in \mathcal{C}(X)$, let $T_{C}$ be the connected component of $T$ that contains $C$. We can define the characteristic polynomial of $\mathcal{C}(X)$ as

$$
\chi(q) \doteq \sum_{C \in \mathcal{C}(X)} \mu\left(T_{C}, C\right) q^{\operatorname{dim}(C)} .
$$

In order to relate this polynomial with $M_{X}(x, y)$, we prove the following fact. Let us assume that $X$ does not contain $\underline{0}$. For every $C \in \mathcal{C}(X)$, set

$$
\mathcal{D}(C) \doteq\left\{A \subseteq X \mid C \text { is a connected component of } H_{A}\right\} .
$$

\section{Lemma 5.5.}

$$
\mu\left(T_{C}, C\right)=\sum_{A \in \mathcal{D}(C)}(-1)^{|A|} .
$$

Proof. The proof is by induction on the codimension of $C$. If it is 0 or 1 , the statement is trivial. Otherwise, by the inductive hypothesis,

$$
\mu\left(T_{C}, C\right)=-\sum_{D \supsetneq C} \mu\left(T_{C}, D\right)=-\sum_{D \supsetneq C} \sum_{A \in \mathcal{D}(D)}(-1)^{|A|} .
$$

Proving that this sum is equal to the claimed one amounts to proving that

$$
\sum_{D \supseteq C} \sum_{A \in \mathcal{D}(D)}(-1)^{|A|}=0 .
$$

Now, let $B$ be the largest (hence minimum with respect to reverse inclusion) element of $\mathcal{D}(C)$. Every $A \in \mathcal{D}(D)$ for $D \supseteq C$ is a subset of $B$, and conversely every $A \subseteq B$ 
is in $\mathcal{D}(D)$ for exactly one $D \supseteq C$ (if there were two such layers $D$, their union would be connected). Therefore

$$
\sum_{D \supseteq C} \sum_{A \in \mathcal{D}(D)}(-1)^{|A|}=\sum_{A \subseteq B}(-1)^{|A|}=0
$$

where the last equality is an elementary combinatorial fact, which is checked by looking at the binomial coefficients of $(1-1)^{k}$.

Theorem 5.6.

$$
(-1)^{n} M_{X}(1-q, 0)=\chi(q) .
$$

Proof. By definition we must prove that

$$
(-1)^{n} \sum_{A \subseteq X} m(A)(-q)^{n-r(A)}(-1)^{|A|-r(A)}=\sum_{C \in \mathcal{C}(X)} \mu\left(T_{C}, C\right) q^{\operatorname{dim} C} .
$$

We remark that

$$
\operatorname{dim}(C)=n-r(A) \forall A \in \mathcal{D}(C)
$$

and

$$
(n-r(A))+(|A|-r(A))+n \equiv|A|(\bmod 2) .
$$

Thus we have to prove that for every $k=0, \ldots, n$,

$$
\sum_{A \subseteq X, n-r(A)=k} m(A)(-1)^{|A|}=\sum_{C \in \mathcal{C}(X), \operatorname{dim}(C)=k} \mu\left(T_{C}, C\right) .
$$

By Lemma 5.4, each $A$ is in $\mathcal{D}(C)$ for exactly $m(A)$ layers $C$. Then Formula (4) is a consequence of Lemma 4.5, since $(-1)^{|A|}$ appears $m(A)$ times in the sum.

Example 5.7. Take $T=\left(\mathbb{C}^{*}\right)^{2}$ with coordinates $(t, s)$ and

$$
X=\{(2,0),(0,2),(1,1),(1,-1)\}
$$

defining equations:

$$
t^{2}=1, s^{2}=1, t s=1, t s^{-1}=1 .
$$

The hypersurfaces $H_{t^{2}}$ and $H_{s^{2}}$ have two connected components each; $H_{t s}$ and $H_{t s^{-1}}$ are connected (but their intersection is not). The 0 -dimensional layers are

$$
C_{1}=(1,1), C_{2}=(-1,-1), C_{3}=(1,-1), C_{4}=(-1,1) .
$$

Notice that $C_{1}$ and $C_{2}$ are contained in 4 layers of dimension 1 each, while each of $C_{3}$ and $C_{4}$ lies in 2 layers of dimension 1 . Then $\mu(T, C)=-1$ for each of the six 1 -dimensional layers $C$, and

$$
\begin{aligned}
& \mu\left(T, C_{1}\right)=\mu\left(T, C_{2}\right)=-(1-4)=3, \\
& \mu\left(T, C_{3}\right)=\mu\left(T, C_{4}\right)=-(1-2)=1 .
\end{aligned}
$$

Hence

$$
\chi(q)=q^{2}-6 q+8
$$

The polynomial $M_{X}(x, y)$ is composed of the following summands:

- $(x-1)^{2}$, corresponding to the empty set;

- $6(x-1)$, corresponding to the 4 singletons, each giving contribution $(x-1)$ or $2(x-1)$;

- 14, corresponding to the 6 pairs: indeed, the basis $X=\{(2,0),(0,2)\}$ spans a sublattice of index 4 , while the other bases span sublattices of index 2 ; 
- $8(y-1)$, corresponding to the 4 triples, each contributing with $2(y-1)$;

- $2(y-1)^{2}$, corresponding to the whole set $X$.

Hence

Notice that

$$
M_{X}(x, y)=x^{2}+2 y^{2}+4 x+4 y+3 .
$$

$$
M_{X}(1-q, 0)=q^{2}-6 q+8=\chi(q),
$$

as claimed in Theorem 5.6

5.4. Poincaré polynomial. For every $C \in \mathcal{C}_{X}$, let us define

$$
X_{C} \doteq\left\{\chi \in X \mid H_{\chi} \supseteq C\right\} .
$$

Remark 5.8. The set $X_{C}$ defines a hyperplane arrangement in the vector space $V_{C} \doteq V / X_{C}{ }^{\perp}$. Let $\mathcal{L}\left(X_{C}\right)$ be its intersection poset. Let $\mathcal{C}(X, C)$ be the poset of the elements of $\mathcal{C}(X)$ that contain $C$. The map

$$
\begin{gathered}
\psi: \mathcal{C}(X, C) \rightarrow \mathcal{L}\left(X_{C}\right), \\
D \mapsto X_{D}{ }^{\perp}
\end{gathered}
$$

is an order-preserving bijection. Indeed, given $L \in \mathcal{L}\left(X_{C}\right)$, set

$$
A(L) \doteq\left\{\lambda \in X,\left.\lambda\right|_{L}=0\right\} .
$$

Then $\psi^{-1}(L)$ is the connected component containing $C$ of $H_{A(L)}$.

Lemma 5.9.

$$
n b c\left(X_{C}\right)=(-1)^{n-\operatorname{dim}(C)} \mu\left(T_{C}, C\right) .
$$

Proof. By the previous remark,

$$
\mu\left(T_{C}, C\right) \doteq \mu_{\mathcal{C}(X)}\left(T_{C}, C\right)=\mu_{\mathcal{C}(X, C)}\left(T_{C}, C\right)=\mu_{\mathcal{L}\left(X_{C}\right)}\left(V_{C}, X_{C}^{\perp}\right)=\chi_{\mathcal{L}\left(X_{C}\right)}(0)
$$

since $X_{C}{ }^{\perp}$ is the origin in $V_{C}$, and hence the only element of rank 0 . Thus by Theorem 4.3 and Formula (3),

$$
\chi_{\mathcal{L}\left(X_{C}\right)}(0)=(-1)^{n-\operatorname{dim}(C)} T_{X_{C}}(1,0)=(-1)^{n-\operatorname{dim}(C)} n b c\left(X_{C}\right) .
$$

Let $T_{1}, \ldots, T_{h}$ be the connected components of $T$. We denote by $\mathcal{C}(X)_{i}$ the set of layers that are contained in $T_{i}$. This clearly gives a partition of the layers:

$$
\mathcal{C}(X)=\bigsqcup_{i=1}^{h} \mathcal{C}(X)_{i} .
$$

We now give some formulae for the Poincaré polynomial $P(q)$ and the Euler characteristic of $\mathcal{R}_{X}$. We start from a restatement of a result proved in [8, Theorem 4.2] (see also [9, 14.1.5]). In this paper is considered an arrangement of hypersurfaces in a torus, in which every hypersurface is obtained by translating by an element of the torus the kernel of a character. It is clear that the restriction of the arrangement $\mathcal{T}(X)$ on every $T_{i}$ is an arrangement of this kind. Then the cohomology of $\mathcal{R}_{X} \cap T_{i}$ can be expressed as a direct sum of contributions given by the layers of this arrangement, which are the elements of $\mathcal{C}(X)_{i}$. In terms of the Poincaré polynomial $P_{i}(q)$ of $\mathcal{R}_{X} \cap T_{i}$, this expression is:

$$
P_{i}(q)=\sum_{C \in \mathcal{C}(X)_{i}} n b c\left(X_{C}\right)(q+1)^{\operatorname{dim}(C)} q^{n-\operatorname{dim}(C)} .
$$


Thus the Poincaré polynomial of $\mathcal{R}_{X}=\bigsqcup_{i}\left(\mathcal{R}_{X} \cap T_{i}\right)$ is just the sum of these polynomials:

\section{Theorem 5.10.}

$$
P(q)=\sum_{C \in \mathcal{C}(X)} n b c\left(X_{C}\right)(q+1)^{\operatorname{dim}(C)} q^{n-\operatorname{dim}(C)} .
$$

Now we prove:

\section{Theorem 5.11.}

$$
P(q)=q^{n} M_{X}\left(\frac{2 q+1}{q}, 0\right) .
$$

Proof. By definition, we have that

$$
q^{n} M_{X}\left(\frac{2 q+1}{q}, 0\right)=\sum_{A \subseteq X} m(A)(q+1)^{n-r(A)} q^{r(A)}(-1)^{|A|-r(A)} .
$$

We compare this formula with the one in the previous theorem. We have to prove that for every $k=0, \ldots, n$ the coefficient of $(q+1)^{k} q^{n-k}$ is the same in the two expressions. In fact by applying Formula (4) and then Lemma 4.9 we get the claim:

$$
\begin{aligned}
& (-1)^{n-k} \sum_{A \subseteq X, r(A)=n-k} m(A)(-1)^{|A|}=(-1)^{n-k} \sum_{C \in \mathcal{C}(X), \operatorname{dim}(C)=k} \mu\left(T_{C}, C\right) \\
& =\sum_{C \in \mathcal{C}(X), \operatorname{dim}(C)=k} n b c\left(X_{C}\right) .
\end{aligned}
$$

Therefore, by comparing Theorem 5.6 and Theorem 5.11, we get the following formula, which relates the combinatorics of $\mathcal{C}(X)$ with the topology of $\mathcal{R}_{X}$, and is the "toric" analogue of Theorem 4.1.

\section{Corollary 5.12.}

$$
P(q)=(-q)^{n} \chi\left(-\frac{q+1}{q}\right) .
$$

We recall that the Euler characteristic of a manifold can be defined as the evaluation at -1 of its Poincaré polynomial. Hence by Theorem 5.11 we have:

Corollary 5.13. $(-1)^{n} M_{X}(1,0)$ is equal to the Euler characteristic of $\mathcal{R}_{X}$.

Example 5.14. In the case described in Example 5.7. Theorem 5.11(or Corollary 4.12) implies that

$$
P(q)=15 q^{2}+8 q+1
$$

and hence the Euler characteristic is

$$
P(-1)=8=M_{X}(1,0) .
$$

5.5. Number of regions of the compact torus. In this section we consider the compact abelian group dual to $\Gamma$,

$$
\bar{T} \doteq \operatorname{Hom}\left(\Gamma, \mathbb{S}^{1}\right)
$$

where we set

$$
\mathbb{S}^{1} \doteq\{z \in \mathbb{C}|| z \mid=1\} \simeq \mathbb{R} / \mathbb{Z}
$$

For simplicity we assume $\Gamma$ to be a lattice: then $\bar{T}$ is a compact torus; i.e. it is isomorphic to $\left(\mathbb{S}^{1}\right)^{n}$, and every $\chi \in X$ defines a hypersurface in $\bar{T}$ :

$$
\overline{H_{\chi}} \doteq\{t \in \bar{T} \mid \chi(t)=1\} \text {. }
$$




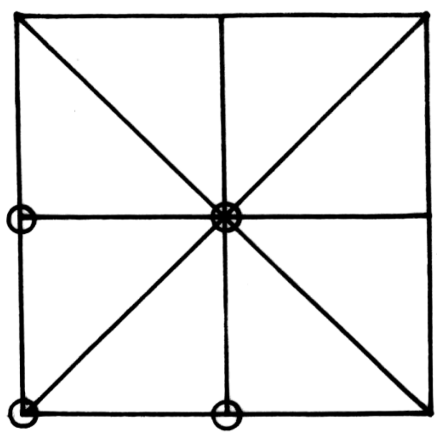

FiguRE 4

We denote this arrangement by $\overline{\mathcal{T}(X)}$. Clearly its poset of layers is the same as for the arrangement $\mathcal{T}(X)$ defined in the complex torus $T$. We denote by $\overline{\mathcal{R}_{X}}$ the complement

$$
\overline{\mathcal{R}_{X}} \doteq \bar{T} \backslash \bigcup_{\chi \in X} \overline{H_{\chi}} .
$$

The compact toric arrangement $\overline{\mathcal{T}(X)}$ has been studied in [12]; in particular the number $R(X)$ of regions (i.e. of connected components) of $\overline{\mathcal{R}_{X}}$ is proved to be a specialization of the characteristic polynomial $\chi(q)$ :

\section{Theorem 5.15.}

$$
R(X)=(-1)^{n} \chi(0) .
$$

By comparing this result with Theorem $[5.6$ we get the following

\section{Corollary 5.16.}

$$
R(X)=M_{X}(1,0)
$$

Example 5.17. In the case of Example 5.7, we can represent in the real plane with coordinates $(x, y)$ the compact torus $\bar{T}$ as the square $[0,1] \times[0,1]$ with the opposite edges identified. Then the arrangement $\overline{\mathcal{T}(X)}$ is given by the lines

$$
x=0, x=1 / 2, y=0, y=1 / 2, x=-y, x=y .
$$

These lines divide the torus in $8=\chi(0)$ regions. See Figure 4 .

\section{DAHMEN-Micchelli SPACES}

So far we considered evaluations of $T_{X}(x, y)$ and $M_{X}(x, y)$ at $y=0$ and $y=$ 1. However, there is another remarkable specialization of the Tutte polynomial: $T_{X}(1, y)$, which (by Theorem 3.6) is called the polynomial of the external activity of $X$. It is related to the corresponding specialization of $M_{X}(x, y)$ in a simple way:

\section{Lemma 6.1.}

$$
M_{X}(1, y)=\sum_{p \in \mathcal{C}_{0}(X)} T_{X_{p}}(1, y) .
$$


Proof. By definition,

$$
M_{X}(1, y)=\sum_{A \subseteq X, r(A)=n} m(A)(y-1)^{|A|-n}
$$

and

But by Lemma 5.4 .

$$
T_{X_{p}}(1, y)=\sum_{A \subseteq X_{p}, r(A)=n}(y-1)^{|A|-n} .
$$

$$
m(A)=\left|\left\{p \in \mathcal{C}_{0}(X) \mid A \subseteq X_{p}\right\}\right|,
$$

which is the number of polynomials $T_{X_{p}}$ in which the summand $(y-1)^{|A|-n}$ appears.

The previous lemma has an interesting consequence. Following [4], 6], and 7], we associate to every finite set $X \subset V$ a space $D(X)$ of functions $V \rightarrow \mathbb{C}$, and to every finite set $X \subset \Lambda$ a space $D M(X)$ of functions $\Lambda \rightarrow \mathbb{C}$. Such spaces are defined as the solutions to a system of differential equations and of difference equations, respectively, in the following way.

For every $\lambda \in X$, let $\partial_{\lambda}$ be the usual directional derivative

$$
\partial_{\lambda} f(x) \doteq \frac{\partial f}{\partial \lambda}(x)
$$

and let $\nabla_{\lambda}$ be the difference operator

$$
\nabla_{\lambda} f(x) \doteq f(x)-f(x-\lambda) .
$$

For every $A \subset X$, we define the differential operator

$$
\partial_{A} \doteq \prod_{\lambda \in A} \partial_{\lambda}
$$

and the difference operator

$$
\nabla_{A} \doteq \prod_{\lambda \in A} \nabla_{\lambda}
$$

We can now define the differentiable Dahmen-Micchelli space

$$
D(X) \doteq\left\{f: V \rightarrow \mathbb{C} \mid \partial_{A} f=0 \forall A \text { such that } r(X \backslash A)<n\right\}
$$

and the discrete Dahmen-Micchelli space

$$
D M(X) \doteq\left\{f: \Lambda \rightarrow \mathbb{C} \mid \nabla_{A} f=0 \forall A \text { such that } r(X \backslash A)<n\right\} .
$$

The space $D(X)$ is a space of polynomials which was introduced in order to study the box spline. This is a piecewise-polynomial function studied in Approximation Theory; its local pieces, together with their derivatives, span $D(X)$. On the other hand, $D M(X)$ is a space of quasi-polynomials which arises in the study of the partition function. We recall that a function $f$ on a lattice $\Lambda$ is a quasi-polynomial if there exists a sublattice $\Lambda_{0}$ of finite index such that $f$ restricted to every coset coincides with a polynomial. The partition function is the function that counts how many ways an element of $\Lambda$ can be written as a linear combination with nonnegative integer coefficients of elements of $X$. This function is a piecewise-quasi-polynomial, and its local pieces, together with their translates, span $D M(X)$.

In the recent book [9] the spaces $D(X)$ and $D M(X)$ are shown to be deeply related with the hyperplane arrangement and with the toric arrangement defined by $X$ respectively. 
In order to compare these two spaces, we consider the elements of $D(X)$ as functions $\Lambda \rightarrow \mathbb{C}$ by restricting them to the lattice $\Lambda$. Since the elements of $D M(X)$ are polynomial functions, they are determined by their restriction. For every $p \in$ $\mathcal{C}_{0}(X)$, let us define the following map:

$$
\begin{aligned}
\varphi_{p} & : \Lambda \rightarrow \mathbb{C}, \\
\lambda & \mapsto \lambda(p)
\end{aligned}
$$

(see Section 2.4.2). In [7] (see also [9, Formula 16.1]) the following result is proved.

\section{Theorem 6.2.}

$$
D M(X)=\bigoplus_{p \in \mathcal{C}_{0}(X)} \varphi_{p} D\left(X_{p}\right) .
$$

Since every $D\left(X_{p}\right)$ is defined by homogeneous differential equations, it is naturally graded, the degree of every element being just its degree as a polynomial. The Hilbert series of $D\left(X_{p}\right)$ is known to be $T_{X_{p}}(1, y)$. In other words, the coefficients of this polynomial are the dimensions of the graded parts (see [3] or 9, Theorem $11.8])$. Then, by the theorem above, the space $D M(X)$ is also graded, and by Lemma 6.1 we have:

Theorem 6.3. $M_{X}(1, y)$ is the Hilbert series of $D M(X)$.

By comparing this theorem with Proposition 2.2 we recover the following known result, which can be found for example in (9, Chapter 13]):

Corollary 6.4. The dimension of $D M(X)$ is equal to the volume of the zonotope $\mathcal{Z}(X)$.

\section{The CASE OF ROOT SYSTEMS}

This section is devoted to describing a remarkable class of examples. We will assume standard notions about root systems, Lie algebras and algebraic groups, which can be found for example in [16] and [15].

Let $\Phi$ be a root system, $\left\langle\Phi^{\vee}\right\rangle$ be the lattice spanned by the coroots, and $\Lambda$ be its dual lattice (which is called the cocharacter lattice). Then we define, as in Section 4.2 , a torus $T=T_{\Lambda}$ having $\Lambda$ as its group of characters. In other words, if $\mathfrak{g}$ is the semisimple complex Lie algebra associated to $\Phi$ and $\mathfrak{h}$ is a Cartan subalgebra, $T$ is defined as the quotient $T \doteq \mathfrak{h} /\left\langle\Phi^{\vee}\right\rangle$.

Each root $\alpha$ takes integer values on $\left\langle\Phi^{\vee}\right\rangle$, so it induces a character

$$
e^{\alpha}: T \rightarrow \mathbb{C} / \mathbb{Z} \simeq \mathbb{C}^{*} .
$$

Let $X$ be the set of these characters. More precisely, since $\alpha$ and $-\alpha$ define the same hypersurface, we set

$$
X \doteq\left\{e^{\alpha}, \alpha \in \Phi^{+}\right\} .
$$

In this way a toric arrangement is associated to every root system.

Remark 7.1. (1) Let $G$ be the semisimple, simply connected linear algebraic group associated to $\mathfrak{g}$. Then $T$ is the maximal torus of $G$ corresponding to $\mathfrak{h}$, and $\mathcal{R}_{X}$ is known as the set of regular points of $T$.

(2) One may take as $\Lambda$ the root lattice (or equivalently, replace the coroot lattice by the character lattice in the construction above). But then one obtains as $T$ a maximal torus of the semisimple adjoint group $G^{a}$, which is the quotient of $G$ by its center. 
Toric arrangements defined by root systems have been studied in [19]; we now show two applications to the present work. Let $W$ be the (finite) Weyl group of $\Phi$, and let $\widetilde{W}$ be the associated affine Weyl group. We denote by $s_{0}, \ldots, s_{n}$ its generators, and by $W_{k}$ the subgroup of $\widetilde{W}$ generated by all the elements $s_{i}$ but $s_{k}$. Let $\Phi_{k} \subset \Phi$ be the root system of $W_{k}$, and denote by $X_{k}$ the corresponding sublist of $X$. Then we have:

\section{Corollary 7.2 .}

$$
M_{X}(1, y)=\sum_{k=0}^{n} \frac{|W|}{\left|W_{k}\right|} T_{X_{k}}(1, y) .
$$

Proof. The proof is straightforward from [19, Theorem 1] and Lemma 6.1

Furthermore, in 19, the following theorem is proved. Let $W$ be the Weyl group of $\Phi$.

Theorem 7.3. The Euler characteristic of $\mathcal{R}_{X}$ is equal to $(-1)^{n}|W|$.

By comparing this statement with Corollary 5.13, we get the following

\section{Corollary 7.4 .}

$$
M_{X}(1,0)=|W| \text {. }
$$

It would be interesting to have a more direct proof of this fact.

Example 7.5. The toric arrangement described in Example 5.7 corresponds to the root system of type $C_{2}$. Notice that the order of the Weyl group of type $C_{2}$ is

$$
8=P(-1)=M_{X}(1,0)=R(X) .
$$

\section{ACKNOWLEDGMENTS}

The author is grateful to his advisor, Corrado De Concini, for many important suggestions. He also wishes to thank Federico Ardila, Michele D'Adderio, Emanuele Delucchi, Matthias Lenz, Mario Marietti, Claudio Procesi, Mimi Tsuruga and Michèle Vergne for stimulating discussions and remarks.

\section{REFERENCES}

[1] F. ARdila, Semimatroids and their Tutte polynomial, Revista Colombiana de Matematicas, 41 (2007) 39-66. MR2355665(2008j:05082)

[2] F. Ardila, A. Postnikov, Combinatorics and geometry of power ideals, Trans. Amer. Math. Soc., 362 (2010), 4357-4384. MR2608410

[3] C. De Boor, N. Dyn, A. Ron, On two polynomial spaces associated with a box spline, Pacific J. Math., 147 (2): 249-267, 1991. MR1084708 (92d:41018)

[4] C. De Boor, K. Hollig, B-splines from parellelepipeds, J. Analyse Math., 42: 99-115, 1982/83. MR729403 (86d:41008)

[5] H. H. Crapo, The Tutte polynomial, Aequationes Math., 3: 211-229, 1969. MR0262095 (41:6705)

[6] W. Dahmen, C. A. Miccheldi, On the solution of certain systems of partial difference equations and linear dependence of translates of box splines., Trans. Amer. Math. Soc., 292(1): 305-320, 1985. MR805964 (86k:41014)

[7] W. Dahmen, C. A. Micchelli, The number of solutions to linear Diophantine equations and multivariate splines, Trans. Amer. Math. Soc., 308(2): 509-532, 1988. MR951619 (90f:11018)

[8] C. De Concini, C. Procesi, On the geometry of toric arrangements, Transformation Groups 10, N. 3-4, 2005. MR2183118 (2006m:32027)

[9] C. De Concini, C. Procesi, Topics in hyperplane arrangements, polytopes and box-splines, Springer, 2010. MR2722776 
[10] C. De Concini, C. Procesi, M. Vergne, Partition function and generalized DahmenMicchelli spaces, arXiv:math 0805.2907.

[11] C. De Concini, C. Procesi, M. Vergne, Vector partition functions and index of transversally elliptic operators, arXiv:0808.2545v1

[12] R. Ehrenborg, M. Readdy, M. Slone, Affine and toric hyperplane arrangements, Discrete Comput. Geom. 41 (2009), 481-512. MR2496314(2010a:52035)

[13] O. Holtz, A. Ron, Zonotopal Algebra, Adv. Math. 227 (2011), 847-894. MR2793025

[14] O. Holtz, A. Ron, Zhiqiang Xu, Hierarchical zonotopal spaces, arXiv:0910.5543v2.

[15] J.E. Humphreys, Linear Algebraic Groups, Springer-Verlag, 1975. MR0396773 (53:633)

[16] J.E. Humphreys, Introduction to Lie Algebras and Representation theory, Springer-Verlag, 3rd reprint, 1975. MR 499562 (81b:17007)

[17] G. I. LeHren, The cohomology of the regular semisimple variety, J. Algebra 199 (1998), no. 2, 666-689. MR1489931 (98k:20080)

[18] M. LenZ, Hierarchical zonotopal power ideals, arXiv:1011.1136 [math.CO].

[19] L. Moci, Combinatorics and topology of toric arrangements defined by root systems, Rend. Lincei Mat. Appl. 19 (2008), 293-308. MR2465681 (2009j:14072)

[20] L. MocI, Geometry and Combinatorics of toric arrangements, Tesi di Dottorato (Ph.D. Thesis), Universitá di Roma Tre, March 2010.

[21] L. MocI, Wonderful models for toric arrangements, arXiv:0912.5461 [math.AG].

[22] J. G. OxLEy, Matroid Theory, Oxford University Press, Oxford, 1992. MR1207587 (94d:05033)

[23] P. Orlik, H. Terao, Arrangements of Hyperplanes, Springer-Verlag, 1992. MR 1217488 (94e:52014)

[24] G. C. Shephard, Combinatorial properties of associated zonotopes, Canad. J. Math., 26: 302-321, 1974. MR0362054 (50:14496)

[25] W. T. Tutre, A contribution to the theory of chromatic polynomials, Canadian J. Math., 6: 80-91, 1954. MR0061366(15:814c)

[26] T. Zaslavsky, Facing up to arrangements: face-count formulas for partitions of space by hyperplanes, Mem. Amer. Math. Soc., 1(154): vii+102, 1975. MR0357135 (50:9603)

Dipartimento di Matematica, "Guido Castelnuovo" Sapienza Universitá di Roma, Piazzale Aldo Moro, 5, 00185, Roma, Italy 